\title{
Deep Ultra-Violet Emission from GaN/AIN Matrix Grown by Plasma-Assisted Molecular Beam Epitaxy
}

Jai Verma, Vladimir Protasenko, S. M. Islam, Amit Verma, Huili (Grace) Xing and Debdeep Jena

Department of Electrical Engineering, University of Notre Dame, Notre Dame, IN 46556, USA

III-V nitride semiconductors are direct band gap semiconductors spanning a wide range of band gaps from $0.7 \mathrm{eV}$ (InN, IR), through $3.4 \mathrm{eV}(\mathrm{GaN}, \mathrm{UV})$ to $6.2 \mathrm{eV}$ (AlN, deep UV). This makes them uniquely suited for fabricating visible and UV LEDs. UV LEDs have applications in water purification, microscopy and chemical analysis. However, as the Al content in the active region increases to access higher energies, several factors like the valence band asymmetry, threading dislocation densities, quantum confined Stark effect, and inefficient $n$ - and p-type doping severely affect the internal quantum efficiency (IQE) of the device. Though, IQE has been increased from $20 \%$ to $60 \%$, contributing to EQE of $10 \%$, by reducing the TD density below $2 \times 108 \mathrm{~cm}^{-2}$ in UV LED structures [1].

Recently, we had shown deep UV emission from GaN/AIN active region grown by plasma assisted MBE [2]. In this work we explore the effect of growth conditions on the peak emission wavelength from GaN/AlN active region. 2 monolayer (ML) thick GaN quantum wells (QWs) and GaN quantum dots (QDs) samples were grown in metal rich regime (III/N $>1)$ and nitrogen rich regime $(\mathrm{III} / \mathrm{N}<1)$, respectively, with AIN as the barrier. Commercially available AlN templates on sapphire have been used for growth. A substrate thermocouple temperature $\left(\mathrm{T}_{\mathrm{c}}\right)$ of $730^{\circ} \mathrm{C}$ is used for all the layers. With $220 \mathrm{~W}$ plasma power a growth rate of 0.173 monolayer (ML)/s is obtained. A $50 \mathrm{~nm}$ AlN nucleation layer is first grown to move away from the template surface. 8 period GaN/AlN layers are then grown with the last GaN layer left uncapped. Fig 1(a) shows streaky 1 x 1 reflection high energy electron diffraction (RHEED) pattern for GaN QWs implying smooth surface. The spotty RHEED pattern for GaN QDs implies a rough surface with $3 \mathrm{D}$ islands. The $2 \times 2 \mu \mathrm{m}^{2}$ atomic force microscope (AFM) scans (Fig $1(c),(d))$ corroborate the smooth and rough surface for QWs and QDs, respectively.

Fig 2 shows the room temperature photoluminescence spectra from the 2 samples. 2 ML thick GaN QWs emit at $272 \mathrm{~nm}$ whereas the $2 \mathrm{ML}$ thick GaN QDs emit a peak wavelength of $248 \mathrm{~nm}$. The change in peak wavelength is attributed to the change in lateral size which in turn affects the confinement and quantization in the GaN layer. Besides, the emission intensity decreases as the wavelength decreases. This implies that the GaN volume emitting the photons has decreased corroborating our inference about decrease in lateral size of GaN leading to a blue shift. However, it is remarkable that emission at $248 \mathrm{~nm}$ $(\sim 5.0 \mathrm{eV})$ can be obtained from quantum confinement of $\mathrm{GaN}$ which has a bulk bandgap of $3.4 \mathrm{eV}$. Fig 3(a) shows the Z-contrast Scanning Transmission Electron Micrograph (STEM) of GaN QWs. We can observe the continuous GaN layers embedded in AlN matrix whereas in Fig 3(b) we observe broken GaN layers for GaN QDs embedded in AlN matrix which is an indication of GaN islands. The ability to control the peak emission wavelength from GaN layers emitting in deep UV by changing the growth parameters provides an excellent platform to utilize GaN/AIN active regions in tunnel injection based UV LEDs with high efficiency.

\section{References:}

[1] M. Shatalov, W. Sun, A. Lunev, X. Hu, A. Dobrinsky, Y. Bilenko, J. Yang, M. Shur, R. Gaska, C. Moe, G. Garrett, and M. Wraback, Appl. Phys. Exp., 5, 082101, (2012).

[2] J. Verma, P. Kandaswamy, V. Protasenko, A. Verma, H. Xing, D. Jena, Appl. Phys. Lett., 102, 041103, 2013 

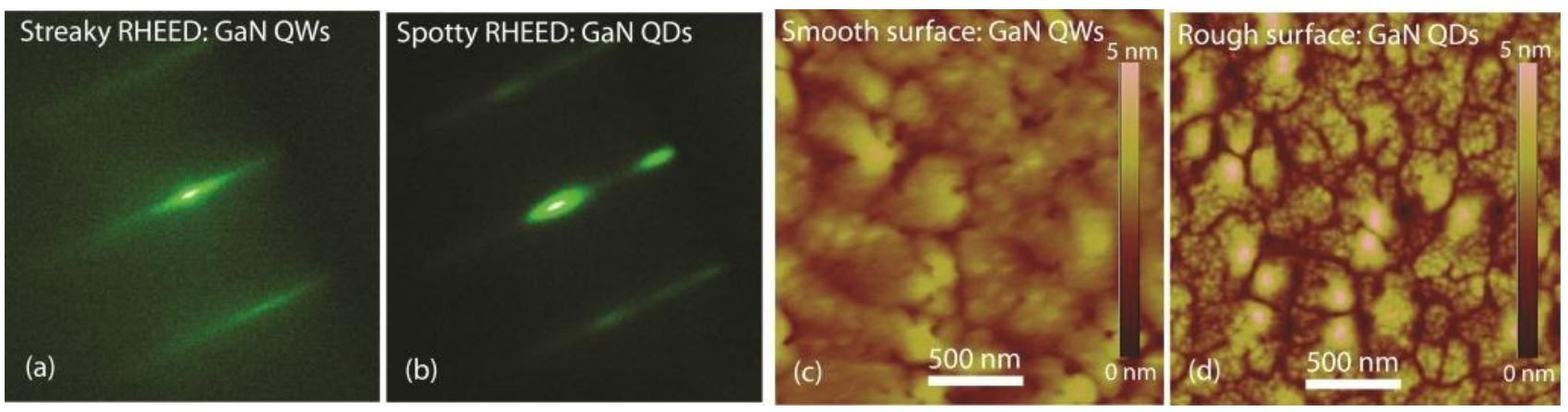

Fig 1. (a) Streaky RHEED pattern for GaN QWs signifying smooth surface; (b) Spotty-streaky RHEED pattern for GaN QDs signifying island formation; (c) Smooth surface shown by AFM for GaN QWs; (d) 3D islands visible on the surface by AFM for GaN QDs

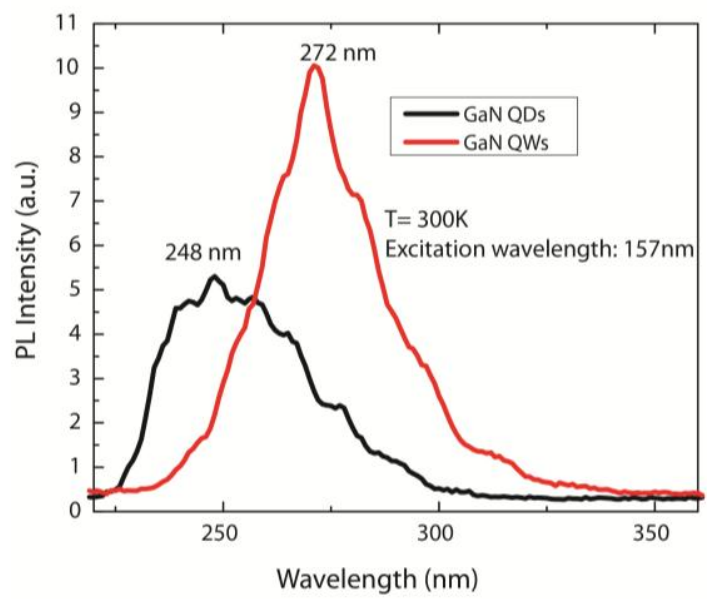

Fig 2. Room temperature PL spectra for GaN QWs and QDs. $2 \mathrm{ML}$ GaN QWs emit at $272 \mathrm{~nm}(4.56 \mathrm{eV})$ whereas $2 \mathrm{ML}$ thick GaN QDs emit at $248 \mathrm{~nm}(5 \mathrm{eV})$. The change in lateral dimensions affects the confinement of carriers leading to higher energy emission from GaN QDs compared to GaN QWs
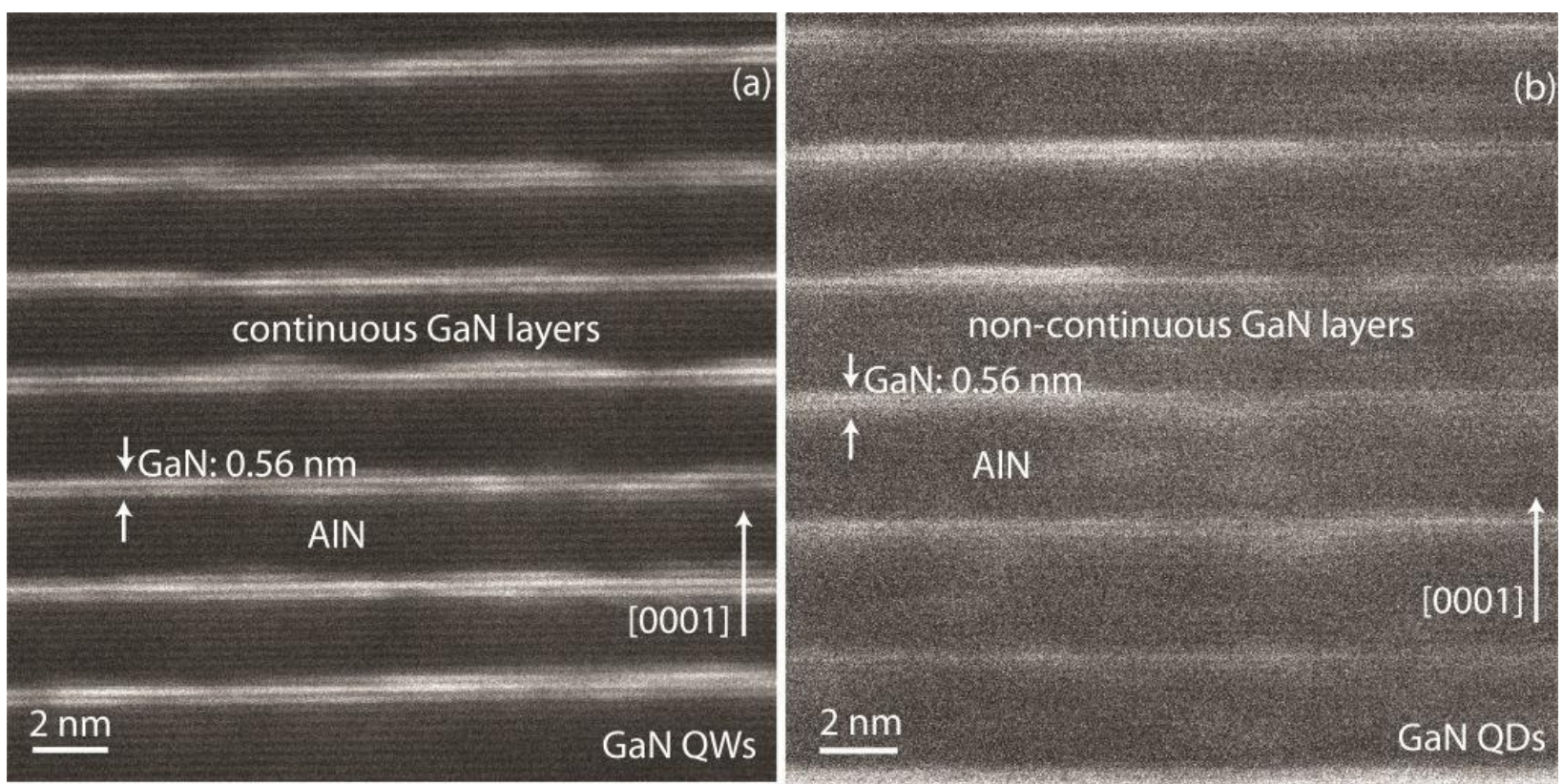

Fig 3. (a) Z-contrast STEM micrograph for GaN QWs showing continuous 2 ML thick GaN layers; (b) STEM micrograph for GaN QDs showing broken GaN layers 